METAL HOMEOSTASIS

\section{Pumping iron}

Science 356, 608-616 (2017)

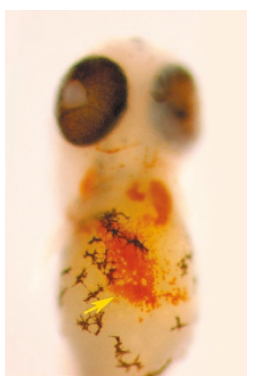

Iron homeostasis requires membrane transporters such as DMT1, mitoferrin, and ferroportin to modulate the movement of iron into and out of cells. Disorders such as anemia are correlated with loss of these transporters, and there are no existing chemical means of restoring transporter function. To address this gap, Grillo et al. used a yeast strain that was defective in the iron-transporting complex FetFtr1 to perform a chemical screen for smallmolecule compounds that could mimic the activity of protein channels and restore iron efflux. The natural product hinokitiol, previously known to function as an iron chelator, restored growth in this mutant strain and also rescued iron transport in cell lines deficient in DMT1, mitoferrin, or ferroportin. UV-visible spectroscopy titration analysis revealed that hinokitiol binds iron with high affinity, and hinokitiol treatment of iron-loaded DMT1-deficient cells, which exhibit a buildup of labile iron, promoted iron efflux. Conversely, hinokitiol promoted iron uptake in the presence of high extracellular iron concentrations. Together, these results suggested that the iron flux effects of hinokitiol are proportional to cellular iron gradients. Finally, hinokitiol promoted gut iron absorption and rescued anemia in transporter-deficient animal models, offering an intriguing new tool for restoring iron transport.

SYNTHETIC BIOLOGY

\section{Playing favorites}

Nat. Commun. 8, 15128 (2017)

Synthetic genetic circuits have allowed researchers to rewire cells, opening the door to broad applications in biotechnology, agriculture and medicine. However, these circuits impose a burden on the host cell and compete with endogenous processes for resources. To address these issues, Venturelli et al. recruited the bacterial endoribonuclease MazF, which recognizes an 'ACA' sequence in single-stranded RNA found in over $96 \%$ of Escherichia coli transcripts, to divert resources to a protected synthetic genetic circuit by degrading transcripts that contain MazF recognition sites. Recoding the $m$ Cherry reporter or glucose dehydrogenase $g d h$ transcripts to lack MazF recognition sites ensures protection from degradation after MazF induction, resulting in enhanced fluorescence or gluconate production, respectively. By building a model of cellular resource allocation, the group revealed the strength of the MazF negative feedback loop and the effectiveness of the stoichiometric inhibitor MazE. Transcriptional profiling of MazF-induced cells uncovered coldshock regulatory elements that were
CIRCADIAN REGULATION

\section{Switching periods}

Mol. Cell. 66, 447-457 (2017)

The bHLH-PAS transcription factor BMAL1 (brain and muscle ARNT-like 1) is a circadian activator that ensures proper periodicity. BMAL1 activity is mediated by the direct interaction of transcriptional activators such as CBP-p300 and the PER-CRY feedback repressors. In particular, CRY1 and p300 compete for binding at the transactivation domain (TAD) to dictate circadian periodicity; mutations in this region can alter the period length. Using NMR analysis of the TAD C-terminal region, Gustafson et al. found that the W624-P625 imide bond, which is conserved in other BMAL1 homologs, undergoes isomerization between cis and trans conformations. Mutation of either W624 or P625 to alanine or substitution of W624 with tyrosine to decrease aromaticity locked BMAL1 in the trans conformation, resulting in a shorter period. Conversely, replacing P625 with a bulky proline analog trapped the cis isomer. In wild-type BMAL1, the isomerization between cis and trans was very slow and required the presence of peptidyl-prolyl isomerases such as cyclophilins to accelerate this conversion; inhibition of cyclophilin using cyclosporine A also increased the circadian period. As the cis and trans isomers exhibited similar binding to CBP and CRY, further studies will be needed to reveal the mechanism by which bond isomerization alters periodicity. upregulated in response to MazF activity. Recruiting MazF as a global regulator of transcript stability enabled alteration of resource allocation inside a host cell. The researchers envision that resource allocation control could be used in biotechnological applications by modulating the balance of cell growth and production in a dynamic manner.

ANTIMICROBIALS

\section{Targeting fungal chromatin Nat. Commun. 8, 15482 (2017)}

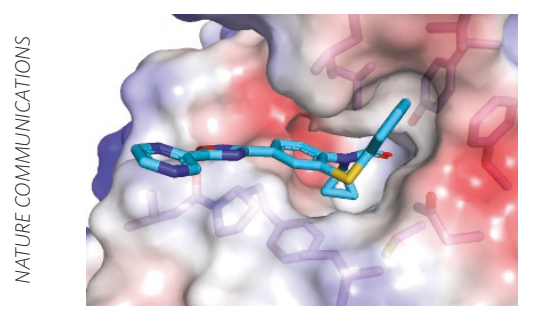

Fungal infections are a major source of complications and morbidity in immunocompromised individuals, with limited treatment options available. Mietton et al. therefore sought to establish whether targeting Candida albicans Bdf1 (CaBdf1) - a member of the BET family of bromodomain (BD)-containing proteins that functions by binding acetylated histone tails-might have therapeutic potential. First the authors established that the two BDs within $\mathrm{CaBdf1}$ are essential for viability in vitro and for virulence in a mouse model of infection. They then showed that $\mathrm{CaBdf1}$ BDs were resistant to BET inhibitors effective against human $\mathrm{BDs}$. The crystal structures of $\mathrm{CaBdf1} \mathrm{BD} 1$ and $\mathrm{BD} 2$ revealed features within their binding pockets divergent from those of human BDs, rationalizing the resistance to existing inhibitors and suggesting that selectivity toward $\mathrm{CaBdf1}$ was achievable. The authors then screened a library of small molecules using a homogeneous time-resolved fluorescence assay to detect disruption of a BD's interaction with tetra-acetylated $\mathrm{H} 4$ peptides. The screen identified a dibenzothiazepinone scaffold with activity toward $\mathrm{CaBdf} 1 \mathrm{BD} 1$ and an imidazopyridine compound active toward $\mathrm{CaBdf1} \mathrm{BD} 2$, but not their human counterparts. These findings suggest that selective BET inhibitors are a potential new class of antifungal therapeutics. 